\title{
Genetic Algorithm Based Node Fault Detection and Recovery in Distributed Sensor Networks
}

\author{
Lokesh B. Bhajantri \\ Department of Information Science and Engineering, \\ Basaveshwar Engineering College, Bagalkot, Karnataka, India - 587102. \\ E-mail: lokeshcse@yahoo.co.in \\ Nalini. $\mathbf{N}$ \\ Department of Computer Science and Engineering, \\ Nitte Meenakshi Institute of Technology, Bangalore, Karnataka, India- 560064. \\ E-mail: nalinaniranjan@ hotmail.com
}

\begin{abstract}
Sensor nodes are prone to failure due to energy depletion and some other reasons in Distributed Sensor Networks (DSNs). In this regard fault tolerance of network is essential in distributed sensor environment. Energy efficiency, network or topology control and faulttolerance are the most important issues in the development of next-generation DSNs. This paper proposes a node fault detection and recovery by using Genetic Algorithm (GA), when some of the sensor nodes faulty in DSN. The main objective of this work is to provide fault tolerance mechanism, which is energy efficient and responsive to network by using GA which is used to detect the faulty of nodes in the network based on the energy depletion of node and link failure between nodes. The proposed fault detection model is used to detect faults at node level and network level faults (link failure and packet error). We have evaluated the performance parameters for the proposed scheme.
\end{abstract}

Index Terms-Distributed Sensor Networks (DSNs), Genetic Algorithm (GA), Fault Detection and Recovery.

\section{INTRODUCTION}

A DSN is a high level distributed set of sensors that are interconnected by a communication network in the environment. The sensors are deeply embedded devices that are integrated with a physical environment and capable of acquiring signals, processing the signals, communicating, context aware computing and performing simple computation tasks. While this new class of networks has the potential to enable wide range of applications, it also pose serious challenges like routing, data gathering and dissemination, frequent network topology change, and fault tolerance[1].

With all these constraints an efficient and effective method to extract faults from the network is challenging task and also pose many new challenges compared with traditional networks as follows: power aware and energy efficient, exception free and unattended operation or self configuring, and must respond to dynamic environment, data-centric and data concentrated and application specific, and fault tolerance [2]. DSNs are often deployed in hostile and unattended environments. Sensors may fail from impact of deployment, fire or extreme heat, animal or vehicular accidents, malicious activity, or by extended use. These failures may occur upon deployment or over time after deployment, extensive operation may drain some of the nodes power or external factors may physically damage part of the nodes. Additionally, hazards may change devices positions over time, possibly disconnecting the network. Any of these initial deployment errors, sensor failures, or change in sensor positions cause the network to be disconnected or lack other desired properties and need to deploy additional sensors to fix the network. It may exist at hardware layer, software layer, network communication layer, node level and application layer [3]. There are various levels of faults in DSN environment such as: at node level or sink node level and network level faults (link failure and packet error). In this work, it has been considered that a node level fault and replace those nodes in the network.

\section{RELATED WORKS}

Some of the related works are given below: A survey on fault management in wireless sensor networks is presented in [4]. This paper addresses the challenge by surveying existing fault management process and also summarizes the existing management architectures. The work presented in [5] depicts a distributed fault tolerant topology control in static and mobile wireless sensor network. In this work a distributed algorithm for assigning minimum possible power to all the nodes in the wireless sensor network was discussed. The work given in [6] presents a distributed topology control in wireless sensor networks with asymmetric links. It considers the problem of topology control in a network of heterogeneous wireless devices with different maximum transmission ranges. The work given in [7] presents a Bayesian decision model for intelligent routing in sensor networks. In this paper, new efficient energy-aware routing algorithm based on learning patterns is presented. The probabilistic decision model both considered the 
estimation of the available energy at the neighboring nodes and the importance of the messages to make intelligent decisions. The work given in [8] describes the selection of cluster heads in wireless sensor networks using Bayesian network. In this paper a Bayesian network based approach is used to select cluster heads. The approach incorporates energy level and signal strength of each sensor node. Experiments have been conducted to compare the performance of the proposed approach and LEACH deterministic cluster head selection and the results shows that the Bayesian network based approach performs better than LEACH deterministic cluster head selection and chain-based LEACH.

The work given in [9] addresses fault-tolerant topology control in a heterogeneous wireless sensor network. It introduces the k-degree any cast Topology Control (kATC) problem. The work given in [10] presents a distributed Bayesian algorithm for fault-tolerant event region detection in wireless sensor networks. It presented two Bayesian algorithms, the randomized decision scheme and the threshold decision scheme, and derived analytical expressions for their performance and proposed solution is in the form of Bayesian fault-recognition algorithms that exploits the measurement errors due to faulty equipment are likely to be uncorrelated, while environmental conditions are spatially correlated.

Bayesian fusion algorithm for inferring trust in wireless sensor networks is presented in [11]. This paper introduces a new Bayesian fusion algorithm to combine more than one trust component (data trust and communication trust) to infer the overall trust between nodes. Simulation results demonstrate that a node is highly trustworthy provided that both trust components simultaneously confirm its trustworthiness and conversely, a node is highly untrustworthy if its untrustworthiness is asserted by both components.

The work in [12] describes a dynamic routing control based on a genetic algorithm. This paper demonstrates that a dynamic routing control based on a genetic algorithm can provide flexible real-time management of dynamic traffic changes in broadband networks. It proposes a string structure, each of whose elements represents paths between each pair of origin and destination terminal nodes, and a new technique using the past solutions as the initial data for new searches. These techniques dramatically improve the efficiency and convergence speed of the genetic algorithm.

The description of genetic algorithm for hierarchical wireless sensor networks is in [13]. In which, hierarchical clusters can reduce the energy consumption requirements for WSNs, investigate intelligent techniques for cluster formation and management. The simulation result shows that the proposed intelligent hierarchical clustering technique can extend the network lifetime for different network deployment environments.

The work presented in [14] describes GA inspired routing protocol for wireless sensor networks. This paper proposes a Genetic algorithm inspired Routing Protocol (GROUP) which shows enhanced performance in terms of energy efficiency and network life time over other schemes.

The work presented in [15] describes genetic algorithm based efficient routing scheme for multicast networks. Due to the advent of many new multimedia applications in high speed networks, the issue of multicast routing has become more and more important. This paper proposes a new multicast routing scheme based on GA.

Fault node recovery algorithm for a wireless sensor networks is presented in [16]. This paper proposes a fault node recovery algorithm for wireless sensor network based on the grade diffusion algorithm combined with a GA. In this work, they have evaluated the performance in terms of active nodes, rate of data loss and energy consumption.

The work given in [17] describes the recovery of faulty cluster head sensor by using GA. In this paper, cluster nodes recovery algorithm has been presented. It can consider recovery of each node based on distance of each node from cluster heads, remained energy of cluster head and member of each cluster head and selects the best cluster head to recovery. Some of the related works are given in [18, 19, 20, 21, 22 and 23].

In this work, there is no cluster head mechanism elected because most of the works on the basis of cluster heads. Because each round cluster head changes in the network and its takes more end to end delay. In order to reduce consuming energy to communicate with sink node, several routing techniques have been suggested which are multi-hop and aware of energy level of nodes in the network. These techniques have overhead due to route detection and to achieve fault tolerance with sink node. Prevent this overhead, unbalanced consumption of energy, and fault tolerance of the network.

In this work, it has proposed that GA inspired fault tolerance protocol for fault detection and recovery over the scheme. For discovering the faulty nodes from the network to increase the efficiency in terms of fault tolerance, time computation, network overhead, successful packet delivery ratio and energy efficiency. In this work, it has considered that two point crossover processes in the proposed scheme but earlier works considers only one point crossover which is less efficient. The following are the important assumptions made in the proposed scheme.

- Nodes are stationary and deployed in a random fashion.

- It is assumed that all nodes in the network are static and have initial energy.

- All nodes are equipped with GPS. During set up phase all nodes convey its location.

- Nodes sense the data periodically and send it to the sink node with multi-hop communication over the network by using GA inspired routing protocol.

The rest of the paper is organized as follows: A proposed heuristic technique is discussed in section III. 
Section IV describes on proposed system model for proposed scheme, fault detection, fault recovery and algorithm of proposed work. Simulation and results analysis are presented in section $\mathrm{V}$ and finally we conclude the proposed work in section VI.

\section{PROPOSED HEURISTIC TECHNIQUE}

This heuristic is used to generate useful solution for optimization, fault tolerance and search problems. GA belong to the larger class of evolutionary algorithms, which generate solutions to optimization problems using techniques inspired by natural evolution, such as inheritance, selection, crossover, and mutation [24]. Traditionally, solutions are represented in binary as strings of 0 's and 1 's, but other encodings are also possible. Node based encoding method is used in the proposed work.

The evolution usually starts from a population of randomly generated individuals and happens in generations. In each generation the fitness of every individual is evaluated from the population, multiple individuals are stochastically selected from the current population (based on their fitness) and modified (recombined and possibly randomly mutated) to form a new population. The new population is used in the next iteration of the algorithm. Commonly, the algorithm terminates when either a maximum number of generations has been produced or a satisfactory fitness level has been reached for the population. When the algorithm is terminated, once may have got the fitness is some for few iterations (i.e. positive solution). Some of the operations used in the proposed GA are as follows:

\section{A. Genetic Representation}

A chromosome is a collection of genes and each chromosome represent a number of nodes or genes in the given network. Chromosome of the proposed GA consists of sequences of positive integers that represent the ID's of nodes. The variable length of the chromosome is represented as the total number of nodes or genes in the network. The length of the chromosome should not exceed the maximum length (total number of nodes in the network).

\section{B. Population $(P)$}

A population is a collection of chromosomes, where a family of ' $r$ ' chromosomes is represented as $\mathrm{P}=(\mathrm{c} 1, \mathrm{c} 2 \ldots$ $\mathrm{cr}$ ). In which as the number of nodes increases, then size of the chromosome structure increases. For the initial population the number of chromosomes structures is generated with presence of faulty nodes. Selection of two chromosomes is based on roulette wheel selection for finding better chromosomes for fault tolerance in the network. Fig. 1 shows a chromosome representation for a network of 500 nodes. In which gene index that represent the position of the nodes (as shown as 1, 2, 3, 4, 5, 6, 7, 8, $9,10 \ldots .$.$) and gene value provides the nodes$ identification number (ID).

\begin{tabular}{|c|c|c|c|c|c|c|c|c|c|c|}
\hline 1 & 2 & 3 & 4 & 5 & 6 & 7 & 8 & 9 & 10 & $\cdots$ \\
\hline 8 & 6 & 7 & 12 & 15 & 4 & 12 & 7 & 10 & 20 & $\ldots$ \\
\hline 8 & 6 & 7 & 12 & 15 & 4 & 12 & 7 & 10 & 20 & $\ldots$ \\
\hline 9 & 7 & 10 & 81 & 23 & 457 & 34 & 46 & 66 & 70 & $\ldots$ \\
\hline 81 & 6 & 77 & 121 & 154 & 164 & 12 & 102 & 110 & 197 & $\ldots$ \\
\hline 58 & 89 & 54 & 220 & 150 & 47 & 124 & 436 & 70 & 20 & $\ldots$ \\
\hline 10 & 60 & 77 & 12 & 15 & 44 & 42 & 87 & 90 & 150 & $\ldots$ \\
\hline 12 & 60 & 77 & 12 & 15 & 44 & 42 & 87 & 90 & 150 & $\ldots$ \\
\hline 2 & 3 & 4 & 12 & 315 & 454 & 46 & 67 & 93 & 100 & $\ldots$ \\
\hline 213 & 16 & 7 & 12 & 15 & 4 & 417 & 18 & 491 & 425 & $\ldots$ \\
\hline 83 & 106 & 117 & 126 & 315 & 224 & 212 & 117 & 210 & 220 & $\ldots$ \\
\hline 88 & 106 & 107 & 128 & 159 & 4 & 12 & 7 & 210 & 420 & $\ldots$ \\
\hline
\end{tabular}

\begin{tabular}{|l|l|l|l|l|l|l|l|l|l|l|}
\hline 108 & 106 & 107 & 112 & 115 & 114 & 112 & 27 & 107 & 206 & $\ldots$ \\
\hline
\end{tabular}

Fig 1. Chromosome Structure.

\section{Fitness function}

Each chromosome is evaluated by fitness function. The proposed fitness function is designed to increase the fault tolerance of the system. It preserves the historically obtained best chromosomes with fitness value. In this work the fitness function is considered as the following parameters to recover each node in the network on the basis of energy, and link efficiency, which eliminates the faulty nodes from the network. The energy of each node should be more than threshold level energy ( $\mathrm{THL}_{\mathrm{EN}}$ ), and the link efficiency between the nodes will be more than threshold level link efficiency $\left(\mathrm{THL}_{\mathrm{eff}}\right)$. The fitness function of the proposed scheme is formulated as follows:

Fitness

(Chromosomes $)=\Sigma_{6=1}^{6} \mathrm{EN} * \Sigma_{1=1}^{6}$ Leff $*$ Path $*$ Fault

Where,

$\mathrm{EN}=$ Energy of each node (Joules)

Leff $=$ Link Efficiency

Path $= \begin{cases}1000 & \text { if path exists } \\ 1 & \text { otherwise }\end{cases}$ 
Path is a variable, which depends upon whether there is an existing path between source and destination or not but there is no cycle in the path.

$$
\text { Fault }=\left\{\begin{array}{l}
\text { Fault }=-1 \\
\text { Not Fault }=1
\end{array}\right.
$$

The fitness should be non negative; otherwise there is a presence of one or more faulty nodes in the network.

Link efficiency can be computed as follows. Let ' $\mathrm{Ci}$ ' be capacity of a discrete-time discrete-valued channel, ' $\mathrm{B}$ ' be the bit rate of a channel, ET be the total energy consumed for transmission of a bit in link i, SNR be the signal-to-noise ratio [25]. Capacity of channel " $\mathrm{i}$ ", is computed by using (2):

$$
\mathrm{C}_{\mathrm{i}}=\mathrm{B} \log _{2}(1+\mathrm{SNR})
$$

Let 'EN' be the energy consumed for the transmission of a bit per distance 'di'. 'E' can be computed by using (3) [25].

$$
\mathrm{E}=\mathrm{EN} * \mathrm{di}
$$

Let 'Leff' be the link efficiency for the nodes in the network. 'Leff' can be computed by using (4) [26].

$$
\text { Leff }=\mathrm{C}_{\mathrm{i}} / \mathrm{E}
$$

Table1 presents the simulation parameters for link efficiency for a network considered for analyzing the scheme.

Table1. Energy vs. Channel Capacity.

\begin{tabular}{|l|l|l|l|}
\hline Energy & Low & Fair & High \\
\hline Min & & & \\
\hline Fair & 0.40 & 0.56 & 0.70 \\
\hline Max & 0.55 & 0.74 & 0.85 \\
\hline
\end{tabular}

\section{Selection}

Selection process is used to select two parent chromosomes which are having lowest fitness value. This selection process can be implemented by many algorithms such as tournament selection, rank selection, steady state selection and roulette wheel selection. The proposed work by use of roulette wheel selection method it gives contribution of each chromosome to the total fitness value. The chromosome with lowest fitness values for recover the faulty nodes and transmitted to crossover pool is considered the proposed work.

\section{E. Crossover}

The two-point crossover $\left(\mathrm{C}_{\mathrm{T}}\right)$ method is used to create new chromosomes. Two individual chromosomes are chosen from the mating pool or population with lowest value to produce two new offspring. Crossover process exchanges the genes of two parent chromosomes. In which, generate random numbers between node1 and $S_{N}$, which is replace this random value in random position. At the random positions to select the nodes from the parents. Choose a crossover point and from that point cross the parent path. First part of first parent is exchanged with first part of second parent. Similarly, first part of second parent is exchanged with first part of second parent. It has been considered that two - point crossover with crossover probability $\left(\mathrm{C}_{\mathrm{P}}\right)$ is between 0.6 and 1.0. Then the genes of this range are displayed in each two chromosomes as shown in Fig.2.

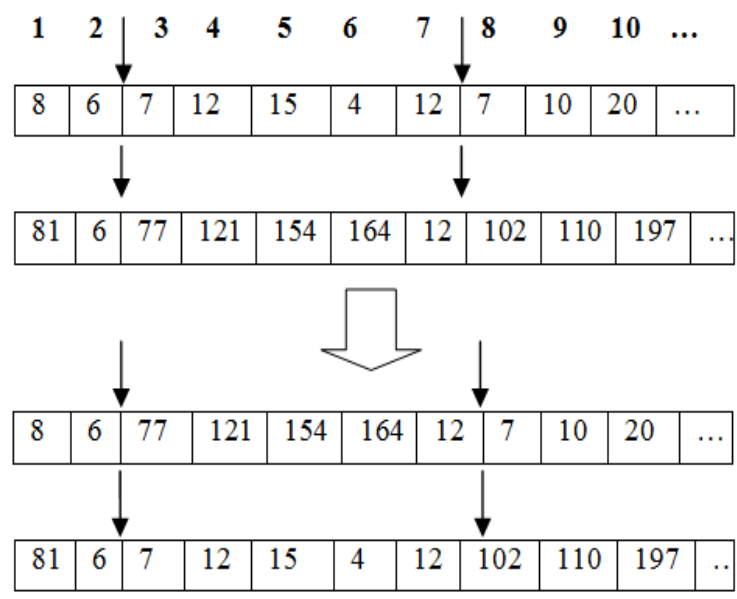

Fig 2. Two Point Crossover.

\section{F. Mutation}

In this work, it considered that ingle point mutation process, in which generate random value between 1 to $S_{N}$ where, ' $\mathrm{S}_{\mathrm{N}}$ ' is number of nodes. Replace this random value in random position. Mutation acts that first a random number between 1 and the number of nodes is produced, and then this value is placed in a random cell within chromosome as shown in Fig.3. It has considered that mutation probability $\left(\mathrm{M}_{\mathrm{P}}\right)$ is between 0.1 and 0.3 .

Selection, crossover and mutation operations are iterated until it meets the termination criteria condition. In the presented algorithm, this method has been used to stop the termination condition until it will reaches to positive values (i.e. recovery of faulty nodes in each chromosomes) otherwise they would continue. 


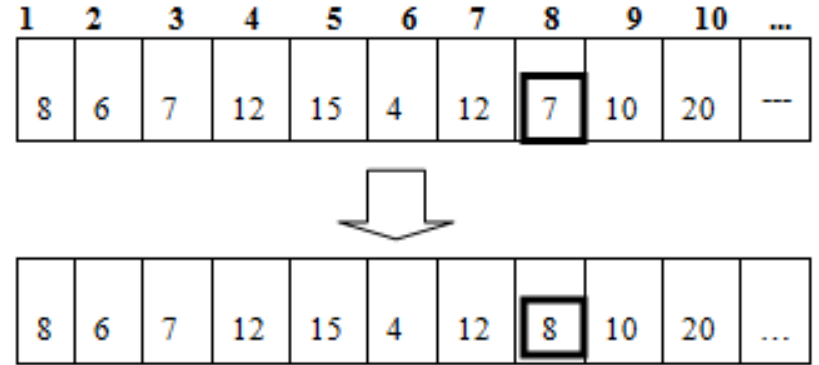

Fig 3. Mutation.

\section{PROPOSED WORK}

Failures are inevitable in DSNs due to inhospitable environment and unattended deployment. Therefore, it is necessary that network failures are detected in advance and appropriate measures are taken to sustain network operation. The work presents an approach to achieve fault tolerance in two phases: 1) fault detection - The fault detection phase is used to find the faulty nodes and the type of faults in the DSN, and 2) fault recovery reinitializes the network to establish path between source nodes and sink node. These two methods are based on GA to achieve fault tolerance in the network.

The proposed GA inspired routing protocol which shows enhanced performance in terms of fault tolerance, fault detection ratio, energy consumption, time complexity, fitness values and packet delivery ratio over the scheme. A multi-path data transfer is also accomplished to provide reliable network operations by considering the fault tolerance of the network with GA. In this section, we describe system model for proposed scheme, fault detection, fault recovery and algorithm of proposed work.

The proposed system architecture consists of set of sensor nodes $S_{N}=\left\{S_{1}, S_{2}, S_{3}, S_{4},-S_{N}\right\}$, which are placed randomly as shown in Fig.4. A node senses the data periodically and sends it to the sink node with multi - hop communication with presence of fault. Sensor nodes are used to compute the fault tolerance in the DSN. The operation sequence of the proposed scheme is as follows: 1) to generate the population size for the network. 2) Select two chromosomes with lowest fitness values based on roulette wheel selection method. 3) Apply genetic operations such as: crossover and mutation operations to two selected chromosomes. 4) To perform fault detection and recovery of each node. 4) To construct the network with active nodes in the DSN environment and 5) Sink node takes the action upon receiving the information from sensor nodes.

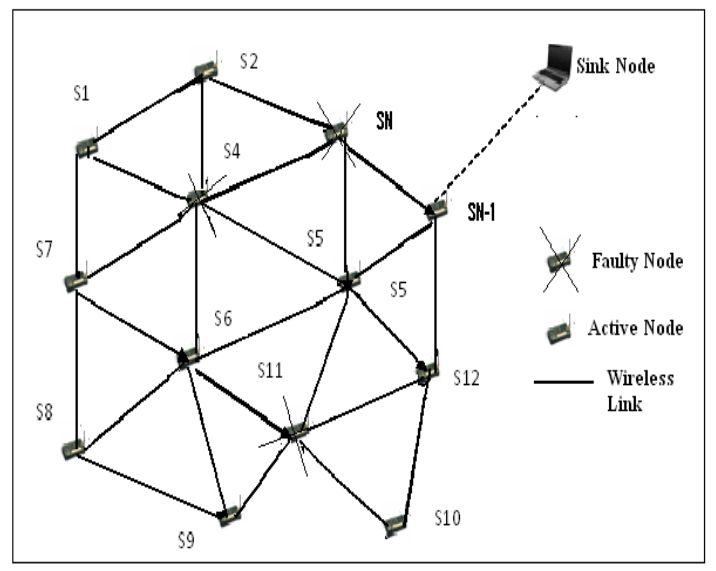

Fig 4. Proposed System Model.

\section{A. Fault Detection}

The fault detection is the initial stage of the DSN, where faults and failures in the network are properly identified in the network using GA. These are the part of the network management system. The aim of the fault detection is to ensure that the services are functioning properly. Since sensor network conditions undergo constant changes, network monitoring alone may not be sufficient to identify network faults. Therefore, fault detection techniques need to be in place to detect potential faults in the network.

\section{B. Fault Recovery}

Fault recovery phase is the stage at which the sensor network is restructured or reconfigured, in such way that failure or faulty nodes to not impact further on network performance. Faulty recovery process is carried out as follows.

- Nodes probability less than threshold: Once fault is detected in the network, the nodes with low probability are sent to sleep mode using GA. This method makes significant improvement in network stability and reduces overhead of network in DSN. The node must be satisfying the following conditions:

$$
\mathrm{S}_{\mathrm{i}} \varepsilon \mathrm{R}_{\mathrm{Si} \leftrightarrow}\left[\left(\mathrm{E}_{\mathrm{Si}} \geq \mathrm{THL}_{\mathrm{EN}}\right) \Lambda\left(\mathrm{R}_{\mathrm{Sj}, \max }>\mathrm{d}_{\mathrm{Si}^{\mathrm{i}}}-\mathrm{S}_{\mathrm{j}}\right)\right]
$$

Where, $\mathrm{Si}$ is a node must be recovered, Rsi is the communication range of sensor node $\mathrm{Si}, \mathrm{RSj}, \max$ is the maximum range of node $\mathrm{Si}$ and $\mathrm{dSi}$-sj is the distance between node $\mathrm{Si}$ and $\mathrm{Sj}$ otherwise sent to sleep mode for fault recovery. For example node 'Si' wants to send packets to node ' $\mathrm{Sj}$ ' first it will measure the fitness of nodes that are in the path between ' $\mathrm{Si}$ ' and sink node. Suppose if any of the nodes probability of energy level is below threshold level probability then it is sent to sleep 
mode and reconstruct the path or network. So, this is used to achieve fault tolerance in the network using GA.

- Link Failure: In case of link failure due to node failure or low link efficiency or bandwidth in network, the next nearest node with probability greater than threshold level probability is selected using GA for forwarding the packets or route the data.

$$
\operatorname{Si} \varepsilon \mathrm{L}_{\mathrm{Si}} \leftrightarrow\left[( \mathrm { E } _ { \mathrm { Si } } \geq \mathrm { THL } _ { \mathrm { EN } } ) \Lambda ( \mathrm { L } _ { \mathrm { Si } } \geq \mathrm { ThL } _ { \mathrm { Leff } } ) \Lambda \left(\mathrm{R}_{\mathrm{Sj}, \max }>\right.\right.
$$

Where, $\mathrm{S}_{\mathrm{i}}$ is a node must be recovered that connect to its neighboring node, $\mathrm{Ls}_{\mathrm{i}}$ is the link efficiency of sensor node $S_{i}, E_{S i}$ is the energy of sensor node $S_{i}$, THL $L_{E N}$ is the threshold level energy of sensor node $S_{i}$, ThL $L_{\text {Leff }}$ is the threshold level link efficiency of sensor node $S_{i}, R_{S j, \text { max }}$ is the maximum range of node $S_{i}$ and $d_{S_{i}}-S_{j}$ is the distance between node $\mathrm{Si}$ and $\mathrm{Sj}$ otherwise node will be faulty or link failure in the network

Link failure between nodes is detected as follows: If node ' $\mathrm{S}_{\mathrm{N}}$ ' does not receive packets from its nearest neighbors, whose probability level is less than threshold within predetermined time interval then it assumes that link from those nodes to node ' $\mathrm{S}_{\mathrm{N}}$ ' are failed.

\section{Algorithm for fault tolerance in DSN Environment}

Begin

1. Deploy the number of nodes in DSN Environment as randomly.

2. Initialize each sensor node properties in the environment such as: sensor_id, energy and link efficiency.

3. Generate initial population which consists of randomly generated chromosomes.

4. Evaluate the quality of each chromosome by using (1).

5. Apply GA operations to proposed scheme as follows:

6. While (1)

- Select two chromosomes with lowest fitness values form the population by using roulette wheel selection approach.

- Apply two-point crossover approach to two selected chromosomes.

- Apply mutation operator to resultant chromosome

- Evaluate the resultant chromosome by using (1).

7. If (fitness for last few iterations is same) \{

8. Stop. (The best chromosome (i.e. positive value of fitness) is to be presented in the network) \} //fault recovery

9. End while // reconstruct the network with active nodes in DSN environment

End
The proposed algorithm (GA) is used to achieve fault recovery of network by evaluating a negative value of fitness to positive value over the nodes in the network.

\section{SimULATION}

The simulation of the proposed scheme is conducted by using ' $\mathrm{C}$ ' programming language. The proposed scheme has been simulated in various network scenarios. Simulation is carried out extensively with random number for 5000 iterations. This section presents the simulation model, simulation procedure, performance parameters, results and discussions.

\section{A. Simulation model}

The proposed simulation model consists of ' $\mathrm{N}$ ' number of nodes deployed randomly in a distributed environment. Simulation is done for ' $N$ ' $(\mathrm{SN}=500)$ nodes used to measure the performance parameters such as probability of fault detection ratio (FDR), probability of fault tolerance, time complexity, energy consumption for fault tolerance, packet delivery ratio (PDR) and fitness values for the proposed scheme. Simulation environment comprises some of the models namely network model, channel model, and battery model. These models are described as follows:

Network Model: It's considered an area of $1 * b$ square meters for DSN environment. Each experiment corresponds to a random placement of sensors in a fixed network area and performs the fault tolerance of network using GA by selecting lowest fitness value for the chromosomes, as it satisfies the fault tolerance criteria.

Channel Model: Sensors generate data packets of variable sizes. S-MAC protocol [27] is used for media access. The transmission of packets is assumed to occur in discrete time. A node receives all packets heading to it during receiving interval unless the sender node is in "non-active", state. For simplicity, it has been considered the channel to be error free.

Battery Model: Each sensor has a battery with finite, non-replenish able energy, which was set to an initial energy in joules. Whenever a sensor transmits or receives a data packet, it consumes some amount of energy.

The energy model for the sensors is based on the first order radio model for calculation of the energy dissipation for sensing and receiving, transmission as well as finding fault tolerance of the network. The radio can perform the power control and hence use the minimum required energy to reach the fault tolerance criteria. It is assumed that at any given time, the value of energy required for transmitted (ET) and sensing (ES) for ' $\mathrm{k}$ ' bit packet to another node ' $\mathrm{d}$ ' meters is 'EN 'Joules for node.

$$
\text { Total Energy }\left(\mathrm{T}_{\mathrm{E}}\right)=\mathrm{E}_{\mathrm{S}} * \mathrm{P}_{\mathrm{k}} / \text { Bits }+\mathrm{E}_{\mathrm{T}} * \mathrm{P}_{\mathrm{k}} / \text { Bits } * \mathrm{~d}_{\mathrm{i}}
$$

Where,

$\mathrm{P}_{\mathrm{k}}=$ Size of packets in terms of bits. 
$\mathrm{d}_{\mathrm{i}}=$ distances between nodes.

\section{B. Simulation procedure}

Table2 presents the simulation parameters considered for analyzing the scheme. Simulation procedure involves following steps:

Begin

1. Deploy the number of nodes randomly as in DSN environment.

2. Initialize sensor node properties.

3. Apply the proposed heuristic technique to networks.

4. Find the fault nodes and recovery of nodes in the network using GA.

5. Compute performance parameters of the system.

6. Generate graphs.

End

Table2. Simulation Input Parameters.

\begin{tabular}{|c|c|c|}
\hline Parameters & Notations & Values \\
\hline Length & 1 & 5000 meters \\
\hline Breadth & $\mathrm{b}$ & 5000 meters \\
\hline Number of Sensor Nodes & $\mathrm{S}_{\mathrm{N}}$ & 500 nodes \\
\hline $\begin{array}{l}\text { Sensor node } \\
\text { communication range }\end{array}$ & $\mathrm{R}$ & 300 meters \\
\hline Selection Type & $\mathrm{S}_{\mathrm{T}}$ & Roulette Wheel Selection \\
\hline Crossover Type & $\mathrm{C}_{\mathrm{T}}$ & Two point \\
\hline Crossover Probability & $\mathrm{C}_{\mathrm{P}}$ & $0.6-1.0$ \\
\hline Mutation probability & $\mathrm{M}_{\mathrm{P}}$ & $0.1-0.3$ \\
\hline Population Size & PS & 500 \\
\hline Number of Iterations & $\mathrm{I}_{\mathrm{N}}$ & 5000 \\
\hline Energy of sensor nodes & EN & 2 Joules \\
\hline Bandwidth per hop & BW & $2 \mathrm{mbps}$ \\
\hline Threshold Level Energy & THL $_{\text {EN }}$ & 0.05Joules \\
\hline $\begin{array}{l}\text { Threshold Level Link } \\
\text { Efficiency }\end{array}$ & THL $_{\text {eff }}$ & $0.297 \mathrm{mbps}$ \\
\hline $\begin{array}{l}\text { Energy required for } \\
\text { sensing of each node }\end{array}$ & $\mathrm{E}_{\mathrm{S}}$ & $50 \mathrm{~nJ} / \mathrm{Bit}$ \\
\hline $\begin{array}{l}\text { Energy required for } \\
\text { transmission of data }\end{array}$ & $\mathrm{E}_{\mathrm{T}}$ & $50 \mathrm{~nJ} / \mathrm{Bit}$ \\
\hline
\end{tabular}

\section{Performance parameters}

The following parameters are used to measure the performance of the proposed scheme as:

1. Probability of Fault Detection Ratio (FDR): As the size of the network increases, decrease in the FDR in the network. It is measured in terms of percentage.

2. Probability of Fault Tolerance: It measures the enables the system to continue to operating properly in the event of failure in DSN environment.

3. Time Complexity: It is defined as the number nodes increases as the percentage of time complexity is increases for the fault tolerance (fault detection and recovery) in the network.

4. Energy Consumption for Fault Tolerance: It is defined as the increase the probability of fault tolerance ratio (FTR) as the decrease the energy consumption for nodes in the network.

5. Packet Delivery Ratio (PDR): It is defined as number of packets sent and receives successfully with increase the number of nodes in the network.

6. Fitness: It is defines as the number of iteration increases, increase in the fault recovery process.

D. Results and Discussions: This section presents the results obtained during simulation.

In Fig.5, the proposed algorithm exhibits probability of fault detection ratio (FDR) with given number of nodes. As the number of nodes increases i.e size of the network increases, decrease in the probability of FDR. The proposed method detects the fault detection efficiently in the network. The size of the network is small, probability of FDR is more; otherwise probability of FDR is gradually decreases. The FDR is measured in terms of percentage.

The Fig.6 shows that as the probability of faulty nodes increases in the network, the probability of fault tolerance of the network decreases. In this work, we have considered the probability of faulty nodes as $25 \%, 50 \%$ and $75 \%$ for the total number of nodes ranging from 100 to 500 . The probability of fault tolerance of the proposed algorithm is more efficient than the other network in the environment.

In Fig.7, the proposed algorithm presents the energy consumption for fault tolerance for varying number of number fault tolerance (\%ge) among nodes. As the number of percentage of fault tolerance increases, there is a less energy consumption of each node in the distributed sensor environment. In this work, we have considered the energy consumption in terms of mJoules. The proposed algorithm utilizes better energy consumption with given number of nodes $\left(S_{N}=100-500\right)$ over the network.

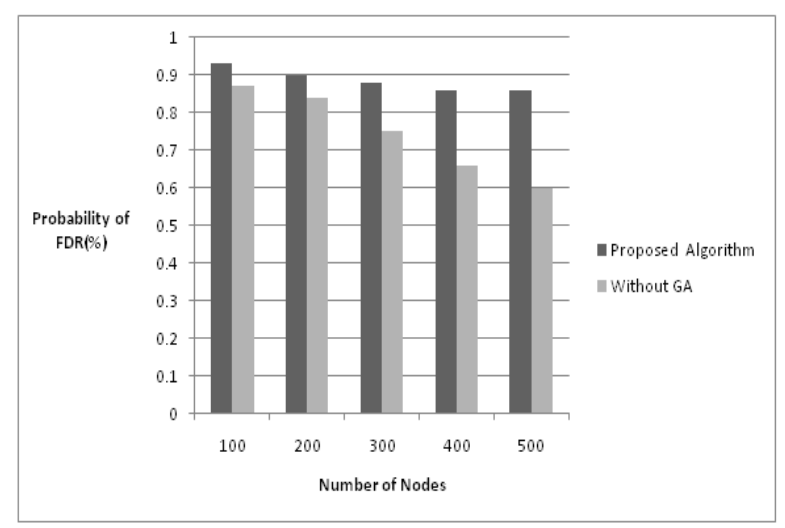

Fig 5. Probability of Fault Detection Ratio (FDR) vs. Number of Nodes. 


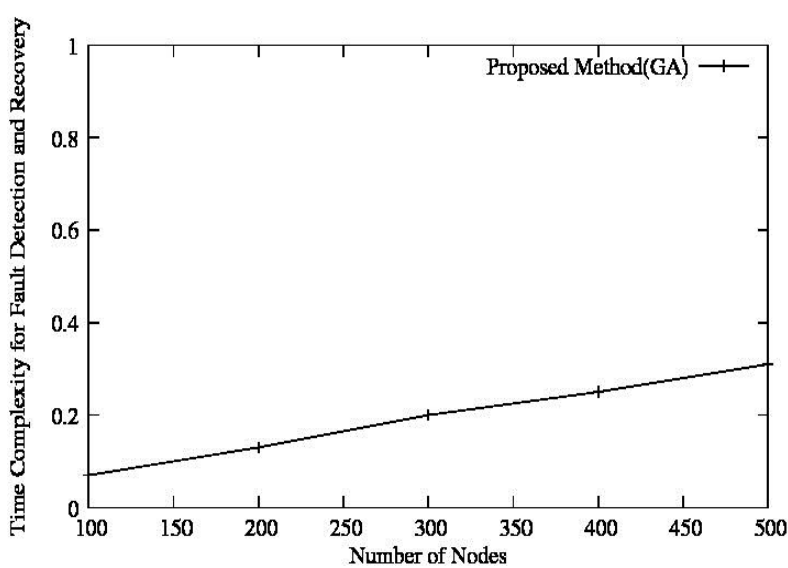

Fig 6. Probability of Fault Tolerance vs. Probability of Faulty Nodes.

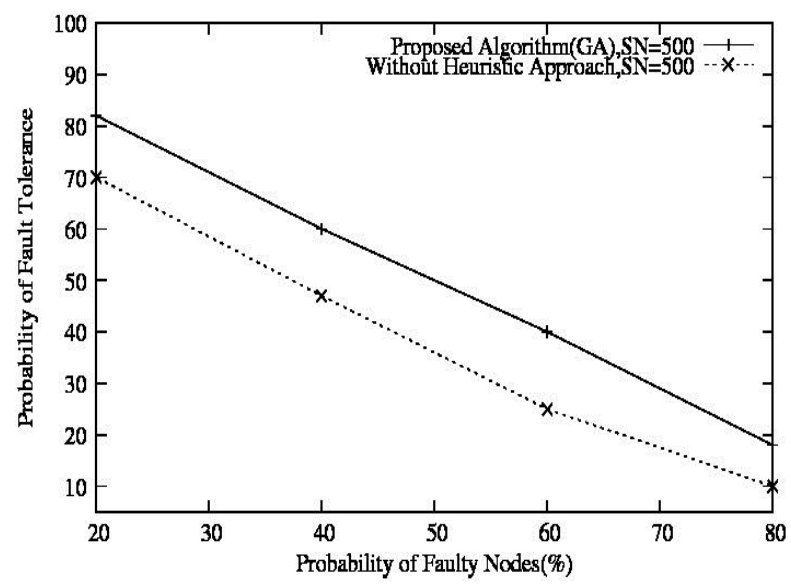

Fig 7.Energy Consumption for Fault Tolerance vs. Number of Nodes.

The Fig.8 shows the time complexity for fault tolerance with given number of nodes in DSN. As the number of nodes increases, fault tolerance complexity increases. With proposed algorithm (GA), time complexity of fault tolerance of the network of DSN will be less time complexity than the without heuristic approach work. In this work, considered that $26 \%$ time complexity proposed method for fault tolerance with given number nodes $(\mathrm{SN}=500)$ in $\mathrm{DSN}$. The time complexity of without use of heuristic network is $\mathrm{O}(\mathrm{n} 2)$. The time complexity of proposed algorithm (GA) is $\mathrm{O}(\mathrm{n} \log n)$ with given number of nodes.

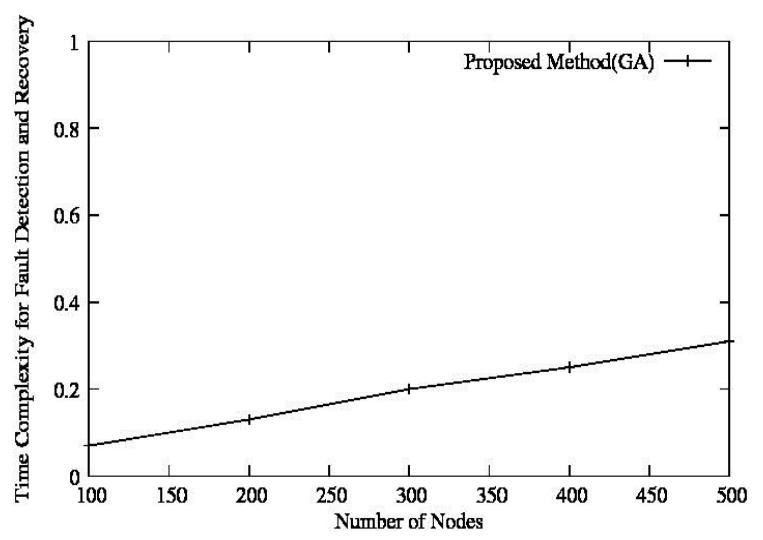

Fig 8.TimeComplexity vs. Number of Nodes.
Fig.9 shows the packet delivery ratio in the DSN environment. As the number of fault tolerance increases, the PDR will be increases in the network with given number of active nodes. In this work, we have considered that $75 \%$ fault tolerance to $92 \%$ of PDR with number of nodes in the network.

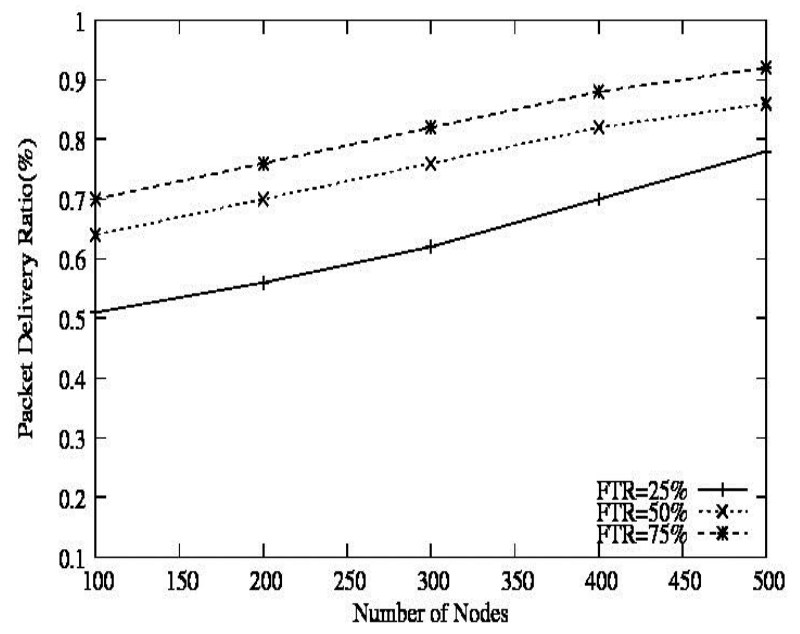

Fig 9. Packet Delivery Ratio (\%) vs. Number of Nodes.

The proposed GA could improve the fitness amount for the chromosomes for some iterations, as Fig.10 shows that produced solutions by initial population have lowest fitness (negative values) value about $-0.0148510^{3}$ (i.e. faulty nodes) and over time and performing iterations this value has been increased gradually and reached to maximum fitness value about $3.82 \times 10^{3}$ for some iterations, which obtained better improvement. Hence we have achieved fault recovery in the network.

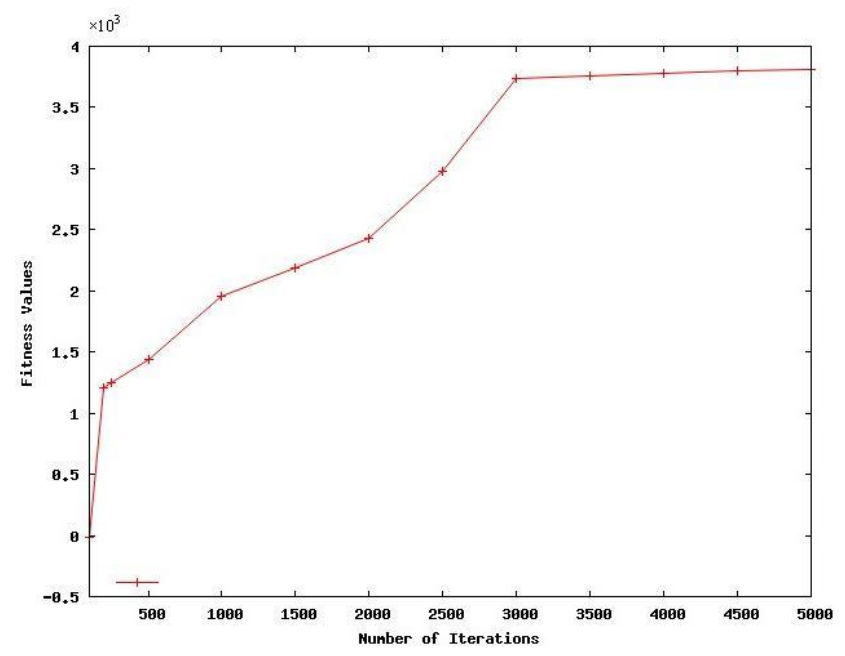

Fig 10. Fitness Values vs. Number of Iterations.

\section{CONCLUSION}

The proposed GA based fault tolerance mechanism is energy efficient and responsive to network in DSN. It includes faults at node level. The proposed system detects energy depletion of a node and link failure between nodes by using GA. 
Simulation results shows that proposed system is more efficient than other network. The following performance parameters analyzed are probability of fault tolerance, time complexity for fault tolerance, network lifetime, energy optimization, fitness values and probability of fault detection rate of the DSN environment. The proposed system performs to continue to operating properly in the event of failure in DSN by using GA. The proposed GA approach is used to generate a solution is quite high in the network.

\section{REFERENCES}

[1] Iyengar. S., S, Ankit. T, Brooks. R., R, An Overview of Distributed Sensors Network. Chapman and Hall/CRC, pp.3-10, 2004. Available: http://books.google.com/books/about/Distributed-sensornetworks.html?id=Nff5.

[2] Al. Karaki J. N. and Kamal, A. E., Routing Techniques in Wireless Sensor Networks: A Survey. Journal on IEEE wireless Communications, vol. 11, pp. 6-28, 2004.

[3] Sitharam S. I, Mohan B., S, Kashyap R., L, Information Routing and Reliability issues in Distributed Sensor Networks, IEEE Transaction on Signal Processing, vol. 40, no. 12, pp. 3012-3021, 1992.

[4] Lilia. P, Qi. H, A Survey of Fault Management in Wireless Sensor Networks. Journal of Network and Systems Management, vol. 15, no.2, pp. 171 -190, 2007.

[5] Mihaela. C, Shuhui. Y, Jie. W, Fault-Tolerant Topology Control For Heterogeneous Wireless Sensor Networks. In the Proceedings of the IEEE International Conference on Mobile Adhoc and Sensor Systems, pp. 1-9, 2007.

[6] Jilei. L, Baochun. L, Distributed Topology Control in Wireless Sensor Networks with Asymmetric Links. In the Proceedings of the IEEE Globecom, Wireless Communications Symposium, vol. 3, pp. 1257-1262, 2003.

[7] Arroyo.V., R, Marques. A., G, Vinagre-Diaz. J, CidSueiro. J, A Bayesian Decision Model for Intelligent Routing in Sensor Networks, In the Proceedings of 3rd International Symposium on Wireless Communication Systems, pp. $103-107.2006$.

[8] Raza. H., A, Sayeed. G, Sajjad. H, Selection of Cluster Heads in Wireless Sensor Networks Using Bayesian Network. In the Proceedings of International Conference on Computer, Electrical, Systems, Science and Engineering, pp. 1- 7, 2010.

[9] Mihaela. C, Shuhui. Y, Jie. W, Algorithms for FaultTolerant Topology Control For Heterogeneous Wireless Sensor Networks. IEEE Transactions on Parallel and Distributed Systems, vol. 19, no. 4, pp. 545 -558, 2008.

[10] Bhaskar. K, Sitharama. I, Distributed Bayesian Algorithms for Fault-Tolerant Event Region Detection in Wireless Sensor Networks, IEEE Transactions on computers, vol. 53, no. 3, pp. 241- 250, 2004.

[11] Mohammad. M, Subhash. C, Rami. A, Bayesian Fusion Algorithm for Inferring Trust in Wireless Sensor Networks, Journal of Networks, vol 5, no 7, pp. 815-822, 2010.

[12] Shimamoto. N, Hiramatsu. A, Yamasaki. K, "A dynamic Routing Control based on a Genetic Algorithm" In the Proceedings of IEEE International Conference on Neural Networks, vol.2, pp.1123 - 1128, 1993.

[13] Sajid. H, Abdul. W., M, Obidul. I, Genetic Algorithm for Hierarchical Wireless Sensor Networks. Journal of Networks, vol. 2, no.5, pp. 87-97 2007.
[14] Ayon. C, SwarupKumar. M, Mrinal. Kanti., N. A Genetic Algorithm inspired Routing protocol for Wireless Sensor Networks. International Journal of Computational Intelligence Theory and Practice, vol, 6, no.1, pp. 1-10, 2011.

[15] Bhattacharya, R. Venkateswaran, P. Sanyal, S.K., Nandi, Genetic Algorithm based Efficient Routing scheme for Multicast Networks. In the Proceedings of International conference on Personal Wireless Communications, pp. $500-504,2005$.

[16] Hong. chi., Shih, Jiun-Huei. Ho, Bin. Yih., Liao, Fault Node Recovery Algorithm for Wireless Sensor Network, IEEE sensors journal, vol.13,no.7, pp. 2683-2689,2013.

[17] Elmira. M. K, Sanam.H, Recovery of Faulty Cluster Head Sensor by using Genetic Algorithm, International Journal of Computer Science Issuues, vol.9.no.1, pp. 141-145, 2012.

[18] Lokesh. B., B, Nalini. N, Energy Aware Based Fault Tolerance Approach for Topology Control in Distributed Sensor Networks, International Journal of High Speed Network, vol. 18, no. 3, pp. 197-210, 2012.

[19] Alaa. F., O, Mohammed. A., 1, Improving the Performance of the Networks Using Genetic Algorithm, In the proceedings of International conference of Advances in Computer Networks and its Security, vol.2, no.3, pp.117-120, 2012.

[20] Myeong.H., L, Yoon.H., C, Fault Detection of Wireless Sensor Networks, Journal of computer communications,vol.31, pp.3469-3475, 2008.

[21] Xiaofeng. H, Xiang. C, Lloyd. E., L, Chien.-Chug. S, Fault-Tolerant Relay Node Placement in Heterogeneous Wireless Sensor Networks, IEEE Transactions on Mobile Computing, vol.9, no.5, pp. 643-656, 2010.

[22] Biao. C, Ruixiang. J, Kasetkasem. T, Varshney. P., K, Channel aware decision fusion in wireless sensor networks, IEEE Transaction on Signal Process, vol. 52, no.12, pp.3454-3458, 2004

[23] Darrell. Whitley, "A Genetic Algorithm Tutorial”. Journal of statistics and computing, vol.4, pp. 65-85, 1994.

[24] Rongbo. Z, Efficient fault-tolerant event query algorithm in distributed wireless sensor networks, International Journal of Distributed Sensor Networks, vol. 2010. no.1155, pp.1-7, 2010.

[25] Herbert. T, Donald. L, Schilling principles of communication, systems. McGraw-Hill 1986.

[26] Miao. G, Himayat. N, Li. G., Y, Energy efficient link adaptation in frequency-selective channels. IEEE Transaction on Communication, vol.58, no.2, pp. 2010.

[27] Heinzelman. W, Chandrakasan. A, Balakrishnan. H. Energy efficient communication protocol for wireless micro sensor networks. In the Proceedings of the IEEE Hawaii international conference on system sciences, vol.8, pp. 8020-8030, 2000.

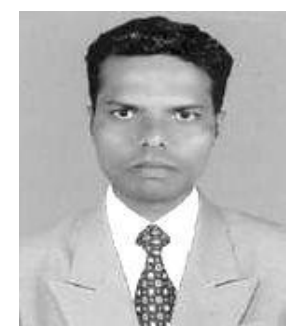

B. Bhajantri Lokesh received his M.Tech. Degree in Computer Science and Engineering (CSE) from Basaveshwar Engineering College, Bagalkot, India, in 2005. He is working as a Assistant Professor in the Department of Information Science and Engineering, Basaveshwar Engineering College, Bagalkot, India. Currently he is pursuing Ph.D. in CSE, Visvesvaraya Technological University (VTU), Belgaum, Karnataka, India. He has 
experience of around 10 years in teaching and research. His areas of interest include Distributed Sensor Networks, eCommerce, u-Commerce, mobile computing and communications, networking protocols, genetic algorithms, applications of agents and real time systems. He has published one book chapter in Handbook of Research on Telecommunications Planning and Management for Business, 8 referred international conferences papers and 7 referred international journals. $\mathrm{He}$ is a reviewer of some journals and conferences. He is a member of Board of Studies (BOS) in the Department of Information Science and Engineering, Basaveshwar Engineering College, Bagalkot, Karnataka, India. $\mathrm{He}$ is a member of International Association of Computer Science and Information Technology (IACSIT).

Department of Information Science and Engineering, Basaveshwar Engineering College, Bagalkot, India. E-mail: lokeshcse@yahoo.co.in

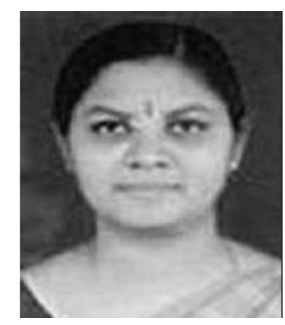

N. Nalini received her Ph.D. from Visvesvaraya Technological University (VTU), Belgaum, Karnataka, India. She is currently working as a Professor, Department of Computer Science and Engineering, Nitte Meenakshi Institute of Technology (NMIT), Bangalore, Karnataka, India. She has experience of around 22 years in teaching and research. She is involved in research of wireless and Distributed Sensor Networks, cloud computing, Cryptography \& Network Security, Genetic Algorithms, and Heuristic Algorithms in Secure Networks. She is an associate editor of Research Journal of Information Technology, Maxwell Scientific Organization. She has many given invited lectures and has conducted several seminars and conferences. She has published 30 in journals and about 50 conferences papers. She is a reviewer of many journals and conferences.

Department of Computer Science and Engineering, Nitte Meenakshi Institute of Technology (NMIT), Bangalore, Karnataka, India. E-mail: nalinaniranjan@ hotmail.com

How to cite this paper: Lokesh B. Bhajantri, Nalini. N,"Genetic Algorithm Based Node Fault Detection and Recovery in Distributed Sensor Networks", IJCNIS, vol.6, no.12, pp.37-46, 2014. DOI: 10.5815/ijcnis.2014.12.05 